\title{
Des poisons au Moyen Âge
}

Dix études sur le venenum et la toxicatio

\section{Franck Collard}

\section{(2) OpenEdition}

Journals

Édition électronique

URL : https://journals.openedition.org/crm/11496

DOI : $10.4000 / \mathrm{crm} .11496$

ISSN : 1955-2424

Éditeur

Honoré Champion

Édition imprimée

Date de publication : 15 juin 2009

Pagination : 1-5

ISSN : 1272-9752

Référence électronique

Franck Collard, «Des poisons au Moyen Âge », Cahiers de recherches médiévales [En ligne], 17 | 2009, mis en ligne le 26 mars 2009, consulté le 15 décembre 2022. URL : http://journals.openedition.org/ crm/11496; DOl : https://doi.org/10.4000/crm.11496

Ce document a été généré automatiquement le 15 décembre 2022.

Tous droits réservés 


\title{
Des poisons au Moyen Âge
}

\author{
Dix études sur le venenum et la toxicatio
}

\author{
Franck Collard
}

Encore largement associée à la brutalité et au sang qui coule, la période médiévale ne vient pas spontanément en premier à l'esprit lorsqu'il est question du poison dans l'histoire. Sans parler de l'époque actuelle, avec l'omniprésence du «toxique » dans l'actualité internationale, politique, écologique et même financière (!), le temps des Césars, la Renaissance ou le Grand Siècle passent pour avoir été plus riches en affaires de poisons que les temps médiévaux, sans doute en raison de leur supposée raffinement supérieur. Même sans succomber au subterfuge immortalisé dans le Nom de la Rose par U. Eco et prêté à Averroès jalousant Avicenne ${ }^{1}$, il suffit pourtant de feuilleter les volumes des chroniques, de Grégoire de Tours à Philippe de Commynes, pour tomber sur du venin. Il suffit de dépouiller un inventaire princier du XIV ${ }^{\mathrm{e}}$ ou du XVe siècle pour trouver objets et ouvrages liés à une hantise de la venenatio, éventuel paravent à une curiosité coupable pour le sujet. Il suffit de parcourir les archives judiciaires laissées par la moindre juridiction pour que surgissent toxicatores et veneficae. Longtemps demeuré dans la sphère de l'anecdote piquante ou de l'énigme historique dont n'avaient que faire les spécialistes de "ce qui s'est réellement passé ", à moins d'avoir été annexé par des médecins-historiens au retro-diagnostic sans appel ou par des chimistes forts de la supériorité de leur temps sur un passé scientifiquement dépassée ${ }^{\text {, }}$ le venenum est aujourd'hui entré dans les territoires du médiéviste, littéraire ${ }^{3}$ ou historien ${ }^{4}$, qu'éclairent les recherches anciennes ou plus récentes des anthropologues ou des ethnologues ${ }^{5}$ sur un « objet » particulièrement intéressant parce que renvoyant à une multitude d'aspects, d'usages, de pratiques et de représentations. Les publications jusqu'ici parues sont fort loin d'avoir épuisé la question. Comme le dit un biographe de Guillaume le Conquérant, réputé, qui l'eût cru en plein âge chevaleresque, empoisonneur patenté, «the subject of medieval poisoning is inexhaustible $»^{6}$. Douglas pense avant tout en termes de nouveaux cas - à l'authenticité variable et souvent invérifiable - qui viennent à jour dès qu'on enquête de manière quelque peu approfondie. Mais la richesse du sujet tient surtout au discours de toute nature et de 
toute origine produit sur le poison et son utilisation dans un monde aux valeurs si contraires à la déloyauté vénéneuse et aux savoirs encore partiellement enchantés.

2 Il reste donc beaucoup à dire et à écrire sur le venenum et la venenatio dans la civilisation de l'Occident médiéval. Signalons d'ailleurs qu'une ouverture du regard vers le monde byzantin et l'aire arabo-musulmane aurait été très profitable au comparatisme et aurait permis de faire encore mieux ressortir l'identité occidentale en la matière. Les propositions de contribution et la place ont manqué. Peut-être le dossier constitué en tirera-t-il davantage de cohérence. Ses auteurs, volontairement choisis parmi les jeunes chercheurs a priori encore à l'abri du ressassement et spécialistes de domaines variés (la littérature latine ou en langues vulgaires, l'histoire de la médecine, l'histoire sociale, l'histoire politique, l'histoire religieuse), proposent dix approches originales et neuves qui peuvent se regrouper autour de quelques thèmes :

- la pensée savante du poison et de la toxicité, entre philosophie naturelle et médecine, grand chantier à peine ébauché pour le Moyen Âge latin quoique L. Thorndike ait montré le chemin voici plus de 70 ans $^{7}$ et alors que d'autres périodes historiques sont pourvues de solides études sur la question ${ }^{8}$;

- la pratique - réelle ou fantasmée - de l'empoisonnement aux marges méridionales ou septentrionales de la chrétienté latine, en des zones dangereusement au contact ou heureusement éloignées des contrées hétérodoxes ou infidèles regorgeant de venena et de toxicatores;

- le crime de poison et sa place dans l'ordre politique et judiciaire qui se met en place à la fin du Moyen Âge à coups de procès et transparaît à travers des représentations littéraires ;

- l'imaginaire métaphorique du poison, image des plus utilisées dans la « communication » religieuse comme dans la littérature.

3 Spécialement novatrices sont les deux contributions sur la pensée savante du poison. L'une aborde la question à travers un traité particulier, le premier du genre en Occident, celui du frère mineur et secrétaire d'Alphonse X Juan Gil de Zamora. L'autre s'interroge plus globalement sur la pensée théorique du venenum dans les textes essentiellement médicaux utilisés ou produits durant le XIV ${ }^{e}$ siècle.

4 C. Ferrero Hernandez reprend le dossier du Contra venena qu'elle a réédité à nouveaux frais en le replaçant non seulement dans l'histoire des textes de philosophie naturelle liés au franciscanisme (frère Juan Gil écrivit aussi une Historia naturalis) comme dans celle des traités "toxicologiques" d'origine arabe, mais aussi dans l'histoire des dissensions internes de l'ordre puisque, suggère l'auteur, la rédaction du traité dédié au ministre général Raimond Geoffroy, proche des spirituels, pourrait bien avoir été suscitée par sa crainte d'un poison "conventuel», sans l'empêcher d'ailleurs, selon certains, d'y succomber en 1310, ce qui relativise d'emblée l'efficacité du genre inauguré par le Castillan.

$5 \mathrm{~J}$. Chandelier propose une synthèse très stimulante sur les mutations de la pensée savante du poison, rattachée à la médecine scolastique au cours du XIV ${ }^{\mathrm{e}}$ siècle par des auteurs lecteurs des Anciens et d'Avicenne, «le premier à faire une place particulière aux poisons dans l'ensemble de sa théorie ». Il forge des concepts destinés à un riche avenir («forme spécifique», "toute substance»), place nettement la science des poisons du côté de l'expérience parce qu'elle est irréductible aux principes, mais réfléchit aussi sur l'articulation entre poison et remède, empoisonnement et maladie. Pietro d'Abano († c. 1316), qui prête aux astres l'origine de la forme spécifique, 
Cristoforo degli Onesti (†1392) et ses Problemata de venenis sont les grands maitres à penser les venena. Le poison a gagné en autonomie à la faveur de spéculations abstraites liées très concrètement à l'épidémie pesteuse qui en a dynamisé, sinon orienté la pensée puisque de multiples passerelles relient la peste au venin.

6 L'exploration d'aires géographiques où le poison prend un relief spécial comprend la Sicile, la péninsule ibérique et, à l'opposé, la Suède. Le cas d'Adélaïde del Vasto met en scène une femme de pouvoir (comtesse de Sicile et Calabre, reine de Jérusalem, mère du futur roi Roger), fort peu appréciée d'Orderic Vital car frottée aux mœurs douteuses de contrées naguère aux mains des Infidèles et encore mal famées à l'époque moderne. Une de ses victimes, Robert de Bourgogne, est identifiée par B. Soustre de Condat.

7 Toujours contiguë aux puissances maures dont la nuisance prend souvent la forme de la toxicatio, l'Espagne est une autre terre de poisons, moins éclairée que l'Italie malgré la publication récente de quelques études de cas'. L'étude très nourrie que F. Ramirès consacre aux poisons chez les Trastamares, dynastie à la légitimité indécise fortement imprégnée de venenum, montre, textes à l'appui, la fréquence des allégations d'empoisonnement dans la société politique castillane qui suit ainsi le modèle des autres sociétés politiques à l'automne du Moyen Âge.

8 Radicalement étrangères aux mœurs méridionales, la Suède constitue un terrain d'enquête complètement neuf et arpenté de façon très efficace par C. Péneau. Si la littérature scandinave présente des histoires très intéressantes (des runes tracés sur une corne à boire emplie de poison la font éclater comme le signe de croix sur la coupe empoisonnée des apôtres), la monarchie élective suédoise ignore largement la venenatio, sauf à la mettre au féminin et au service de la propagande que sainte Brigitte en personne n'hésite pas à déployer. Mais la société le prend en compte, notamment à travers des textes juridiques (Loi nationale de 1442), et sa perception ainsi que ses usages sont finalement assez proches de ce qui prévaut plus au sud de la chrétienté : le crime de poison est le plus horrible de tous, il est lié à la magie et aux femmes (aux pouvoirs inquiétants car subvertissant l'ordre mâle), il entretient un rapport ambigu avec la violence et vient souvent de l'extérieur, du voisin danois ou d'une princesse de France contre qui se déchaînèrent les passions au XIV siècle.

9 Ces enquêtes en terres excentrées montrent majoritairement les liens entre pouvoirs et poisons, même si l'exemple suédois vient rappeler le profond enracinement social d'un crime largement partagé. Au cœur de la chrétienté latine, le poison participe à l'émergence d'un ordre politique en construction, non tant parce qu'il ouvre le chemin des trônes que parce qu'il sert de motif de réflexion sur l'autorité ou de motif (plus ou moins justifié) pour l'imposer. L'étude d'H. Bellon concernant l'épisode si riche de sens de l'empoisonnement (prétendu) d'Alexandre dans la littérature française donne une utile synthèse sur un thème très célèbre dont elle montre l'équivocité (titre d'indignité pour le mort sans combattre ou de gloire pour le guerrier invincible?), les enseignements politiques (orgueil et démesure vaincus, exercice solitaire du pouvoir sanctionné, dont la soif est fatale) et les récupérations (mort de l'empereur Henri VII en 1313) par une littérature mêlant l'histoire et la fiction. Le poison mortel humanise et héroïse à la fois tout en rappelant au puissant la précarité de toute gloire terrestre.

10 Il sert aussi d'élément explicatif aux disgrâces. Dans sa relecture de l'affaire Jacques Cœur, amorcée par la charge d'empoisonnement commis contre Agnès Sorel, dont les restes ont récemment parlé (du mercure mais pas de crime), P. Prétou entend montrer la forte cohérence symbolique des accusations portées contre l'argentier du roi. 
Assurément, le venenum qui se serait répandu dans le corps de la Dame de Beauté comme celui qui, sous forme de fausse monnaie, se serait écoulé dans le royaume, ont une parenté entretenue par les accusateurs, d'autant que la pratique de l'alchimie dont on a accusé aussi le créancier de Charles VII n'est pas sans rapport avec le mélange des substances par lesquelles on obtient du poison. Sans doute peut-on lire la mission du procureur Dauvet comme une sorte de purification du corps du royaume infecté par les finances toxiques de l'homme d'affaires, grand falsificateur, comme une thériaque administrée au royaume à reconstruire, de même que l'on rétablit la santé des empoisonnés par de puissants évacuatifs. L'auteur va jusqu'à dire englobante, à la place de la lèse-majesté, l'incrimination d'empoisonnement à l'aune de laquelle tout le procès aurait été mené. À tout le moins peut-on relever que venenum se prête remarquablement bien à l'instrumentalisation idéologique et à l'interprétation historique tant il suscite la métaphore et appelle l'analogie. La difficulté est moins de décrypter l'implicite que d'être certain de sa présence.

11 L'ordre royal s'impose aussi par la chasse aux sorcières. N. Ghersi porte son regard sur les réalités méridionales (vues notamment depuis le Parlement de Toulouse) de la persécution qui impute aux sorcières (le mot est en ces lieux uniquement au féminin), là comme ailleurs, mais sans doute plus systématiquement, la pratique de l'empoisonnement. La venefica latine, à la double acception d'empoisonneuse et faiseuse de maléfices, devient en Languedoc la " pousonniere » dont les mixtures comprennent les ingrédients habituels, à bases d'herbes mauvaises et d'animaux venimeux., et dont la répression revêt un enjeu politique entre les juridictions concurrentes ainsi qu'une dimension de purification.

12 Face aux forces du diable et du péché que servent les sorcières et qui se matérialisent si bien dans le poison, l'Église veille. Il n'est pas étonnant que la métaphore toxique pour désigner le mal sous ses diverses formes, dont l'hérésie, ait beaucoup servi, comme «image intentionnelle», aux Pères, aux polémistes et aux prédicateurs. Fl. Chave montre les origines antiques (le serpent est une image bien connue, employée contre les hérésiarques, et saint Augustin a appelé le démon venenator) ainsi que les usages de cette métaphorisation du mal qui sert idéalement à mettre en garde les fidèles contre les séduisantes apparences de nocives doctrines et contre la dissimulation trompeuse des hétérodoxes, tout en permettant de rendre l'idée d'une possession inopinée de l'âme par le péché ou l'hérésie qui la pervertissent, de même que le venenum assimile subrepticement le corps à lui pour le faire périr. L'exorcisme équivaut alors à un évacuatif, bénédictions et confession valent pour thériaques salutaires. Aux infections spirituelles des antidotes de même nature s'imposent.

13 Avec la dernière contribution de $\mathrm{P}$. Levron qui rapproche - suggestivement - plus qu'elle ne croise les sources littéraires du Moyen Âge central et les sources médicales, on en revient au poison comme maladie rapportable à divers dérèglements des humeurs, eux-mêmes responsables de maladies de l'âme qui s'insinuent insidieusement en elle comme un venin dans le corps. Plusieurs héros subissent les lentes atteintes de la mélancolie comme s'ils étaient atteints d'un poison à terme et les littérateurs se complaisent à en décrire les effets auto-destructeurs. Mais les remèdes évoqués par les littérateurs sortent souvent du cadre de la médecine et la thériaque apparaît peu, comme si la circulation des notions entre divers milieux lettrés manquait de fluidité.

14 Ces dix études illustrent la fécondité du poison comme objet de réflexion et comme point d'observation pluridisciplinaire sur le monde médiéval. Comme un puissant 
réactif, il révèle ses valeurs et ses hantises, mais permet aussi d'explorer les rapports de pouvoir ou la construction des savoirs. Il reste encore beaucoup à faire en matière de problemata de venenis. L'examen des aires extra-occidentales serait des plus utiles. L'étude générale des traités des poisons reste à mener. Nous nous y employons. Indiquons enfin, sans prétendre épuiser les chantiers à ouvrir, qu'il manque une exploration minutieuse et systématique de l'iconographie paradoxale du crimen occultum qu'est l'empoisonnement. Les recherches médiévales demeurent donc encore loin de la cauda pour ce qui est du venenum.

\section{NOTES}

1. La légende évidemment totalement anachronique de la tentative d'empoisonnement d'Avicenne par Averroès au moyen d'un ouvrage aux feuillets toxiques a traversé le Moyen Âge. On la trouve dans le Flagellum de Nicolas Jacquier (OFP), ch. 13 ou dans le De medicine claris scriptoribus de Symphorien Champier, Lyon, c. 1506. Nous remercions M. Ostorero d'avoir attiré notre attention sur cette histoire.

2. Voir par exemple L. Lewin, Die Gifte in der Weltgeschichte, Heidelberg, 1920, Cologne, 2000, même si l'auteur juge fausse la formule Dubia etiam pro falsis habenda. E. Gilbert, Essai historique sur les poisons, suivi d'une esquisse sur la pharmacie au Moyen Âge depuis la période arabe, Paris, 1868, indique fièrement dans son introduction: "la chimie n'avait pas encore atteint le degré de perfection qu'elle possède aujourd'hui » et stigmatise p. 42 «l'esprit crédule et superstitieux du Moyen Âge ». L'ouvrage de J. de Maleyssie, Histoire du poison, Paris, François Bourin, 1991, paraît à la croisée des deux tendances, à la fois assez fragile historiquement parlant et épousant le point de vue de la science pharmacologique moderne.

3. Cf. C. Cahné, Le philtre et le venin dans Tristan et Iseut, Paris, 1975.

4. F. Collard, Le crime de poison au Moyen Âge, Paris, PUF, 2003 et Pouvoir et poison. Histoire d'un crime politique de l'Antiquité à nos jours, Paris, Seuil, 2007 ; M. Kintzinger, « Maleficium et veneficium. Gewalt und Gefahr für den Fürsten im französischen Spätmittelalter », Königliche Gewalt - Gewalt gegen Könige. Macht und Mord im spätmittelalterlichen Europa, éd. M. Kintzinger et J. Rogge, Berlin, 2004, p. 71-99.

5. Des poisons: nature ambiguë, $\mathrm{n}^{\circ}$ spécial d'Ethnologie française, 34 (2004-3);C. Boujot, Le venin, Paris, Stock, 2001 (sur le bestiaire venimeux surtout); Ph. de Felice, Poisons sacrés et ivresses divines, Paris, 1936, rééd. 1970 ; E. Perrot, E. Vogt, Poisons de flèches et poisons d'épreuves, Paris, 1913 ; A. Retel-Laurentin, Sorcellerie et ordalies: l'épreuve du poison en Afrique noire, Paris, Anthropos, 1974.

6. D. C. Douglas, William the Conqueror, Londres, 1964, p. 415 n. 3.

7. L. Thorndike, An History of Magic and Experimental Science, Londres, 8 vol., 1923-1958, en particulier t. 3 p. 525-545: ch. XXXI sur les traités des poisons du XIV e siècle, et t. 4, en particulier p. 180 et sq.

8. A. Touwaide, «Les poisons dans le monde antique et byzantin : introduction à une analyse systémique ", Revue d'histoire de la pharmacie, 290, 1991, p. 265-281 et surtout "Galien et la toxicologie » Aufstieg und Niedergang der römischen Welt, 2, 37, 2, Berlin, 1994, p. 1887-1986.

9. Exemple J. M. Nieto Soria, Un crimen en la corte. Caída y ascenso de Gutierre Gómez Álvarez de Toledo, Señor de Alba (1376-1446), Madrid, 2006. 


\section{AUTEUR}

FRANCK COLLARD

Université Paris-Ouest Nanterre 